\title{
Théologiques
}

\section{Crise du travail, crise de la civilisation occidentale}

\section{Michel Beaudin}

Volume 3, numéro 2, octobre 1995

Crise du travail, crise de civilisation

URI : https://id.erudit.org/iderudit/602420ar

DOI : https://doi.org/10.7202/602420ar

Aller au sommaire du numéro

Éditeur(s)

Faculté de théologie de l'Université de Montréal

ISSN

1188-7109 (imprimé)

1492-1413 (numérique)

Découvrir la revue

Citer ce document

Beaudin, M. (1995). Crise du travail, crise de la civilisation occidentale.

Théologiques, 3(2), 5-12. https://doi.org/10.7202/602420ar d'utilisation que vous pouvez consulter en ligne.

https://apropos.erudit.org/fr/usagers/politique-dutilisation/ 


\title{
LIMINAIRE
}

\section{Crise du travail, crise de la civilisation occidentale}

\author{
Michel BEAUDIN \\ Faculté de théologie \\ Université de Montréal
}

\begin{abstract}
Une enquête statistique démontrerait probablement que les deux thèmes-vedettes de l'opinion publique (médias, discours gouvernemental, débats électoraux ou ... référendaires!) sont, d'une part l'emploi et, d'autre part, le taux de croissance économique avec son ombre, l'humeur des marchés financiers. Le premier est la forme qu'a imposée la société moderne au travail humain. Le second désigne ce qui est devenu le principe d'orchestration et d'homogénéisation de cette même société, dite marchande. Or, depuis les années 70 , pour la première fois, ces deux réalités non seulement ne vont plus de pair, mais elles entrent en collision. Le progrès technologique et la croissance ne créent plus d'emplois, mais les détruisent plutôt. Au lieu de la "libération" du et dans le travail, annoncée dans les années 60 , c'est la "privation » de l'emploi et la dégradation du travail qui sont survenues de façon accélérée, et avec elles, la privation de la citoyenneté réelle. Si bien que la dynamique de notre monde apparaît maintenant comme un gigantesque processus de détérioration des conditions de vie et d'exclusion sociale, se développant au rythme même de la fuite en avant de l'économie de marché.
\end{abstract}

La "question sociale " que l'on croyait s'être dissoute dans le sillage de la prospérité rebondit donc brutalement. Les événements semblent confirmer cette conviction du Pape Jean-Paul Il (dans la foulée de tout l'enseignement social de l'Église catholique depuis un siècle) que "le travail est une clé, et probablement la clé essentielle, de toute la question sociale ${ }^{1}$.

1 Laborem exercens, n 3. 
La population pressent spontanément que, de proche en proche, la crise de l'emploi est aussi celle de l'économie, qui devient abstraite, se réduisant à un flux d'ordres d'achat et de vente; qu'elle est aussi celle de la société moderne et peut-être bien de la civilisation occidentale. La trame même de notre organisation collective semble nous échapper et se défaire ou, du moins, obéir à une autre logique que celle du territoire et du lien social. L'ampleur historique de ce drame en fait une question de premier plan pour les sociétés. La revue Théologiques y reconnaît aussi d'emblée un "signe des temps" et donc un lieu d'interpellation radicale pour les chrétiennes et les chrétiens.

Déjà, il y a quelques années, le doyen de la Faculté de l'éducation permanente de l'Université de Montréal, M. Jacques Boucher, sonnait l'alerte: «L'Université ne peut faire abstraction de la crise qui secoue le marché du travail ${ }^{2}$. "Aucune institution prétendant être au service de la société n'a le droit de rester à l'écart. Et c'est aussi en tant que lieu de réflexion universitaire que Théologiques voudrait apporter sa contribution, en dialogue avec d'autres partenaires des sciences sociales et de l'intervention sur le terrain. Moins que jamais, la théologie n'a le droit de verser dans la logologie en réduisant une question aussi grave en une simple occasion d'une démarche intellectuelle close sur elle-même. Les lecteurs et lectrices pourront sans doute constater que le discours proprement théologique occupe une place relativement modeste dans ce dossier et que, même lorsqu'il s'exprime, il reste "ex-centrique ", c'est-à-dire constamment tendu vers l'enjeu même qui nous occupe. Nous avons sciemment exclu toute réflexion sur le "travail " de la théologie comme tel, espérant que l'ensemble du dossier en fournisse une illustration plus probante.

Toute crise majeure invite à une reconsidération des fondements de la situation fragilisée. Le drame de l'emploi, tel qu'il se développe aujourd'hui, nous ramène à un questionnement radical touchant le travail humain qui apparaît à la fois comme "cheville ouvrière" des sociétés et comme une "fenêtre " privilégiée sur leur recherche d'un paradigme nouveau et donc sur leur devenir.

Cette prise de distance est d'autant plus stratégiquement urgente que la dynamique économique et politique néo-libérale, si peu appropriée à la situation, semble se renforcer à la fois dans son fonctionnement autoréférentiel et dans son idéologie anti-utopique absolument paralysante pour les esprits. Les politiciens sont en désarroi et se contentent, les yeux fixés

2 Forum XXVI/27 (30 mars 1992), p. 1 et 6. 
sur le court terme, de "gérer" la dérive, de faire comme si les paramètres n'avaient pas changé et comme s'il fallait simplement attendre qu'une conjoncture plus favorable ramène les choses à la «normale ». Même des traditions intellectuelles habituellement critiques sur ce genre de questions paraissent frappées d'aphasie ou restent à court d'explications et d'utopies crédibles.

C'est de cette dynamique dont j'ai tenté de rendre compte dans le premier article. Partant du ras des questions de tout le monde et des données économiques et politiques disponibles, la première partie cherche à démontrer le caractère structurel de l'évolution récente de l'emploi, modelée par le néo-libéralisme. Dans la seconde partie, prenant acte de la culture du désespoir sécrétée par le néo-libéralisme, j'essaie de débusquer l'arbitraire de l'economically correct que le néo-libéralisme fait peser sur le travail, sur l'économie, sur la société et sur l'imaginaire collectif par l'imposition d'une limite auto-transcendante qui sacrifie ou fait tomber dans l'inexistence ou "l'irréalisme " tout ce qui reste extérieure à cette « clôture ».

Dans le deuxième article, Carlos Heredia, un économiste mexicain engagé dans le mouvement populaire, témoigne du même processus du point de vue du Tiers-Monde, mais avec cette particularité intéressante que son pays se trouve dans la même zone de libre-échange que le Canada. Il insiste avec raison, au départ, sur le facteur aggravant d'une démocratie malade de la présence ininterrompue du même parti au pouvoir depuis 1929. L'article décrit ensuite l'holocauste silencieux du "desempleo" produit par les politiques d'ajustement structurel imposées à des pays comme le Mexique, en guise de remède aux maux du sous-développement. Il permet de juger à sa juste valeur de la prétention du capitalisme que le Tiers-Monde soit en mesure de «profiter » grandement de la libéralisation de l'économie mondiale et pose les conditions d'un renversement de la situation.

Les autres articles du dossier entreprennent ensuite, en autant de directions, d'approfondir la problématique du travail, que les articles introductifs avaient abordée à un niveau essentiellement descriptif. Les deux suivants, ceux de Manfred Bischoff et d'André Gorz mettent en perspective historique et anthropologique le complexe travail-économiesociété actuel.

Le premier se propose de récuser cette idée que l'humanité aurait toujours "travaillé ". Il montre d'abord que la notion de travail n'a acquis une connotation spécifiquement économique qu'à partir du XVII ${ }^{2}$ siècle, ce qui transforme la question en la suivante: «existe-t-il une économie en 
toute société? " Si Marx et les sciences sociales ont critiqué le dogme voulant que le travail ait existé sous sa forme marchande en toute société, ils n'ont cependant pas échappé à une conception de l'économie comme "fait de nature ». D'où leur définition matérialiste du travail comme "activité visant à satisfaire les besoins matériels de l'humanité ", et qui demeure entièrement prisonnière de l'idéologie économique. L'auteur suit ici une autre voie. En comparant les sociétés traditionnelles et les sociétés modernes à partir de leur mode spécifique de régulation et de reproduction des rapports sociaux, il montre que c'est du côté de la normativité et non de la positivité qu'on peut expliquer la constitution d'une sphère objective d'activités appelée "économie " et, conséquemment, l'émergence des sociétés modernes comme des "sociétés de travail". L'idée que des sociétés aient pu exister sans "travail " est profondément troublante et subversive. Ne nous renvoie-t-elle pas à considérer que le "travail" actuel n'a pas seulement à voir avec la liberté mais aussi avec la subordination? ${ }^{3}$

Pour sa part, André Gorz, l'un des principaux penseurs européens depuis des décennies sur les questions du travail et de société, et qui nous fait l'honneur d'une contribution à ce dossier, trace plutôt les chemins de l'avenir à partir d'une analyse démontrant que la "société salariale» en est à son déclin définitif. Au lieu des politiques à courte vue de "l'emploi pour l'emploi " et même de la voie d'un "revenu d'existence ", sources d'une nouvelle dualisation, il plaide pour une mutation politique et culturelle qui déconnecte la continuité du revenu de la continuité de l'emploi et qui fasse reposer le lien social tant sur la prestation par tous d'un travail de moins en moins important que sur l'importance croissante reconnue à d'autres types d'activités. Cette utopie est maintenant techniquement à portée de main. L'auteur avance, en terminant, plusieurs propositions pour parer aux dérapages de la situation actuelle en attendant qu'une véritable politique de redistribution du travail ait porté ses fruits. Mais les sociétés sauront-elles saisir cette chance et contrer les forces qui s'opposent à ce projet où le travail pourrait réintégrer l'« œuvre » du développement personnel et collectif?

En écho à cette réflexion, j'aimerais citer les propos prémonitoires qu'André Gorz signait en 1978, sous le pseudonyme de Michel Bosquet, au terme d'une enquête menée en France :

3 Voir Robert L. HEILBRONER, "The World of Work ", dans Behind the Veil of Economics. Essays in the Wordly Philosophy. New York, W.W. Norton, 1988, p. 80103. 
Si tout le monde prenait conscience qu'il n'y a plus, virtuellement, de problème de production mais seulement un problème de distribution - c'est-à-dire de partage équitable des richesses produites et de répartition équitable dans toute la population du travail socialement nécessaire - le système social actuel aurait de rudes difficultés à se maintenir. Que deviendrait la discipline du travail, l'éthique du rendement, l'idéologie de la compétition, si chacun savait qu'il est techniquement possible de vivre mieux en travaillant de moins en moins, et que le droir à un "plein revenu " n'a plus besoin d'être réservé à ceux qui fournissent un "plein travail»?

Pour que l'ordre actuel ne soit pas sapé dans ses fondements idéologiques, mieux vaut que ces choses ne se sachent pas. On dira donc à la population non pas qu'elle n'aura plus besoin de tant travailler mais que le "travail va manquer"; non que nous aurons de plus en plus de temps libre mais "qu'il y aura moins d'emplois». On présentera les promesses de l'automatisation comme des menaces, on tentera de faire en sorte que les travailleurs se disputent entre eux les trop rares emplois au lieu de lutter ensemble pour une autre rationalité économique ${ }^{4}$.

Un texte du poète québécois Félix Leclerc, "Les emplois fiables", l'un des derniers qu'il ait écrit sur ce thème, critique sous un autre mode la logique néo-libérale du travail, maintenant acculée à l'absurde. Madame Gaétane Leclerc a spontanément accepté que nous l'insérions dans ce dossier.

Le travail est-il libération ou punition ( «à la sueur de ton front») de Dieu? Conscients de l'ambiguïté des positions historiques du judéo-christianisme sur la question du travail et de leur indéniable influence dans le façonnement de notre culture, nous avons invité Aldina da Silva à présenter une vue d'ensemble de la vision biblique du "travail ${ }^{5}$ " et de son destin à travers la tradition chrétienne occidentale. Après quelques utiles considérations étymologiques et un bref rappel du vocabulaire hébraïque et grec désignant le "travail " dans la Bible, l'auteure, appuyée sur une sélection représentative de textes, distingue les conceptions pessimiste et optimiste qui courent à travers l'Ancien et le Nouveau Testament. Elle

4 "Quand les chômeurs seront heureux... », Le Nouvel Observateur, 4 décembre 1978 , p. 82-83.

5 La réflexion de Manfred Bischoff incite maintenant à toutes les prudences sur l'emploi de ce terme pour les sociétés anciennes! 
montre ensuite que, contrairement à ce qu'on trouve dans la Bible, c'est la conception pessimiste, trop liée à quelques textes isolés de leur contexte, qui prédominera dans la tradition chrétienne de l'Occident. Le ton change à compter des années 50 de notre siècle où, sauf exception, le travail se met à faire l'objet d'une appréciation théologique unilatéralement positive, ce qui ne laisse d'interroger dans le contexte actuel de la crise du travail.

Dans une "note" (relativement longue), Pierre Goldberger vient compléter l'article précédent en articulant deux faces moins connues de la question du travail. Convaincu de l'importance, en théologie, de ne pas réfléchir de façon trop abstraite sur le travail, il évoque d'abord l'aggravation des conditions de vie et de travail que le capitalisme néo-libéral fait peser sur la grande majorité des populations du Tiers-Monde et surtout sur l'" invisibilisation" séculaire du travail des femmes malgré son ampleur et son rôle prépondérant. Cetre pénible réalité lui sert de fil conducteur pour retracer, dans sa propre tradition réformée, un filon minoritaire mais constamment vigoureux et faisant contrepoids à la perspective protestante qui "libérait les mains de la bourgeoisie marchande montante" : celui d'un certain soupçon jeté sur le travail, lui retirant la prétention de se donner sa propre justice au bout de ses œuvres, et le réinscrivant dans le règne de la liberté et de la grâce. Cette approche retrouve un écho salutaire dans la théologie contemporaine du sabbat, chez un Jürgen Moltmann, par exemple, qui trace des limites au travail luimême en tant que tel, que celui-ci soit œuvre divine ou œuvre humaine. Dieu n'y est jamais Deus Faber, ni son "image ", réductible à l'homo faber. Le sabbat resurgit à point nommé aujourd'hui comme liberté par rapport au travail et donc comme espérance.

Enfin, un article aux proportions considérables ferme la marche. Il nous vient encore du Tiers-Monde et paraît exemplaire d'une théologie contextuelle pour aujourd'hui, c'est-à-dire capable de prendre pleinement en compte la dynamique sociétale, de se renouveler dans ce mouvement même, et de faire une contribution pertinente aux débats cruciaux en cours, y compris dans leur dimension de confrontation. Son auteur est Franz J. Hinkelammert, l'un des chefs de file de la théologie de la libération en Amérique latine, et avec qui j'ai eu le privilège de travailler quelques semaines en 1993. Un critique l'a présenté ainsi : "économiste par métier, philosophe par la radicalité de son questionnement et théologien par voca- 
tion $^{6}$ ». À notre connaissance, son article est le premier de cette envergure à être publié en français par une revue. Nous pouvons présumer que l'influence de ce penseur va continuer de s'étendre dans le monde francophone comme c'est déjà le cas en Amérique latine, dans le monde anglosaxon et en Allemagne. On sera peut-être surpris de ne pas trouver de référence directe au travail dans son article, mais compte tenu de ce que d'autres ont développé concernant la problématique foncièrement "économique " du travail aujourd'hui, on réalisera rapidement l'intérêt inédit de cette réflexion pour notre thème. On y retrouve un historique original de la théologie de la libération comme telle, mais abordée, ici, en étroite liaison avec le problème de l'économie.

L'article retrace, à travers l'expérience personnelle qu'en a connue l'auteur, la genèse, la trajectoire et le rôle fondamental qu'a joué la question de l'économie au sein de la théologie de la libération. C'est à partir de l'articulation originale de cette théologie à l'économie qu'il rend compte de l'hostilité qu'elle a suscitée de la part de l'orthodoxie ecclésiale et théologique et de la part de l'« orthodoxie » du capitalisme néo-libéral. Il décortique longuement la "théologie" capitaliste qui tente maintenant de se substituer à la théologie de la libération après l'avoir vainement dénoncée. L'article se termine sur une critique théologique acérée de l'absolutisation de la «loi», de l'anti-utopisme et de la sacrificialité inhérents au néo-libéralisme actuel, qualifié de totalitaire. Il est parcouru par une trame fondamentale qui fait l'originalité profonde de la théologie de la libération et que n'acceptera jamais la "théologie " néo-libérale: la reconnaissance mutuelle des sujets humains comme des êtres corporels liés par nature à des nécessités vitales, et qui implique l'option pour les pauvres. Les pauvres sont le lieu où se décide si cette reconnaissance est effective ou non. C'est là que le néo- libéralisme achoppe et se révèle, en fin de compte, faussaire. D'où la tâche de "désoccidentaliser " " le monde dans la mesure où l'Occident s'est identifié à une logique néo-libérale surproductrice de biens mais aussi de conditions infra-humaines et de "goulags 8 " à ciel ouvert dans le Tiers-

6 A. ABASCAL-JAEN "Théologie de la vie et réalité économique ", Liaisons internationales 65 , décembre 1990, p. 2.

7 Pour une analyse des racines de la sacrificialité inhérente à l'économie capitaliste occidentale, voir, du même auteur, La fe de Abraham y le Edipo occidental. San José, DEI, 1989, $101 \mathrm{p}$.

8 Au Mexique, les gens appellent les nouveaux bidonvilles « Friedmantowns », du nom de l'économiste néo-libéral Milton Friedman. 
Monde, anticipation de la trajectoire qu'elle a aussi commencé à imprimer aux sociétés du Nord.

Puisse ce dossier, finalement, nous faire davantage prendre la mesure des enjeux soulevés par la question du travail, conscience de leurs médiations concrètes, et nous inspirer quelque engagement à cet égard. Et pour les croyantes et les croyants, qui percevront avec raison que cette question touche la teneur même du projet de Dieu, je formule le souhait que sa lecture fasse retrouver, peut-être, comme le dit le psalmiste, un "zèle dévorant pour la maison (oikos) du Seigneur ", du Dieu qui est lui-même "Économiste" ", c'est-à-dire qui prend un soin jaloux de sa "maison " qu'est l'humanité, et où son Esprit est toujours à l'« œuvre ».

9 Selon la magnifique expression de M. Douglas MEEKS, God the Economist. Minneapolis, Fortress Press, 1989. 